\title{
Thermal effect of bainitic transformation in tube steels during accelerated cooling
}

\author{
M. L. Lobanov ${ }^{1, \uparrow}$, G. M. Rusakov ${ }^{1,2}$, V.N. Urtsev³, M.L. Krasnov, E. D. Mokshin ${ }^{3}$, \\ A. V. Shmakov ${ }^{3}$, S. I. Platov ${ }^{5}$ \\ †m.l.lobanov@urfu.ru \\ ${ }^{1}$ Ural Federal University, 19 Mira St., Yekaterinburg, 620002, Russia \\ ${ }^{2}$ Institute of Metal Physics, Ural Branch of RAS, 18 S. Kovalevskoi St., Yekaterinburg, 620990, Russia \\ ${ }^{3}$ Ausferr Research and Technology Center, 18 Gorkogo St., Magnitogorsk, 455000, Russia \\ ${ }^{4}$ OJSC Magnitogorsk iron and steel works, 93 Kirov St., Magnitogorsk, 455000, Russia \\ ${ }^{5}$ Nosov Magnitogorsk state technical university, 38 Lenin Ave., Magnitogorsk, 455000, Russia
}

An original laboratory bench, which allows for modeling technological rates of cooling of hot-rolled sheets in the process of controlled thermomechanical processing (TMCP) has been designed. Samples cut from an industrial sheet of 06G2MB type pipe steel used in production of large diameter pipes with strength grade X80 have been used. Time dependencies of the actual temperature at initial cooling rates of $100-500 \mathrm{~K} / \mathrm{s}$ have been obtained. All processing modes resulted in almost identical structures mainly formed due to the bainitic transformation. The dispersion of structures decreased with the increase of the cooling rate. Thermotechnical calculations were carried out assuming that the temperature equalization over the sample thickness was instant. The times of transformation amounted to 1-9 s. Most of the transformation at all cooling modes occurred in conditions close to the isothermal one. The athermal nature of the bainitic transformation in TMCP was recorded. Within the observed interval of cooling rates, the temperature at the starting point of the bainitic transformation was $660-730^{\circ} \mathrm{C}$. The heat effect approximately was equal to $120 \mathrm{~kJ} / \mathrm{kg}$, which is twice larger than the thermal effect of the martensite transformation for low-carbon steels. This fact suggests that the kinetics of the bainitic transformation is largely determined by the energy of the slowest process, namely, the re-arrangement of carbon atoms in the austenite occurring parallelly to the shear transformation of FCC lattice into BCC one. It is assumed that energy contribution of the redistribution process of carbon atoms (if their amount is small) to the thermal effect of the bainitic transformation is comparable, at least, to the energy effect of the shear lattice rearrangement.

Keywords: pipeline steel, thermo-mechanical controlled processing, bainite, bainite transformation, thermal phase transition effect.

УДК: 669.15-194.2:53.06

\section{Тепловой эффект бейнитного превращения в трубных сталях при ускоренном охлаждении}

\author{
Лобанов М. Л. ${ }^{1,}$, Русаков Г. М. ${ }^{1,2}$, Урцев В.Н. ${ }^{3}$, Краснов М. Л. ${ }^{4}$, Мокшин Е. Д. ${ }^{3}$,
}

Шмаков А. В. ${ }^{3}$, Платов С. И. ${ }^{5}$

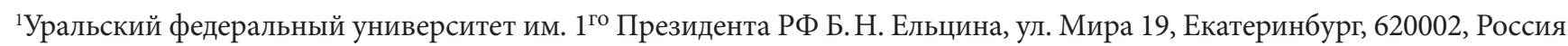
${ }^{2}$ Институт физики металлов имени М.Н. Михеева УрО РАН, ул. С. Ковалевской 18, Екатеринбург, 620990, Россия ${ }^{3}$ ИТЦ «Аусферр», ул. Горького 18, Магнитогорск, 455000, Россия

${ }^{4}$ Магнитогорский металлургический комбинат, ул. Кирова, 93, Магнитогорск, 455000, Россия

${ }^{5}$ Магнитогорский государственный технический университет им. Г. И. Носова, пр. Ленина, 38, Магнитогорск, 455000, Россия

Разработан оригинальный лабораторный стенд, позволяющий моделировать технологические скорости охлаждения горячекатаных листов в процессе контролируемой термомеханической обработки (thermo-mechanical controlled processing - ТМСР). В качестве материалов для исследований использовались образцы, вырезанные из промышленного листа малоуглеродистой низколегированной трубной стали типа 06Г2МБ, предназначенного для производства труб большого диаметра классов прочности К60, К65. Были получены зависимости фактической температуры 
образцов от времени при начальных скоростях охлаждения от 100 до $500 \mathrm{~K} / \mathrm{c}$. При всех режимах термической обработки в образцах были зафиксированы практически идентичные структуры, сформированные преимущественно в результате бейнитного превращения. Средние размеры бейнитных зерен уменьшались приблизительно в два раза с увеличением скорости охлаждения. Теплотехнические расчеты осуществлялись в предположении, что выравнивание температуры по толщине образца происходило мгновенно. В исследуемом интервале скоростей охлаждения температура начала бейнитного превращения составила $660-730^{\circ} \mathrm{C}$. Времена превращения составляют от 1 до 9 с. При всех скоростях охлаждения большая часть превращения реализовывалась в условиях, близких к изотермическим. Зафиксирован атермический характер бейнитного превращения, протекающего в процессе контролируемой термомеханической обработки. Величина теплового эффекта оказалась приблизительно равной 120 кДж/кг, что в два раза превышает тепловой эффект мартенситного превращения для низкоуглеродистых сталей. Этот факт позволяет предположить, что кинетика бейнитного превращения в большой мере определяется энергией самого медленного из процессов, а именно, перераспределения атомов углерода в аустените, происходящего параллельно со сдвиговой перестройкой ГЦК-решётки в ОЦК-решётку Следует полагать, что энергетический вклад процесса перераспределения атомов углерода (при сравнительно небольшом их количестве) в тепловой эффект бейнитного превращения как минимум, сопоставим с энергетическим эффектом сдвиговой перестройки кристаллической решетки.

Ключевые слова: трубные стали, контролируемая термомеханическая обработка (ТМСР), бейнит, бейнитное превращение, тепловой эффект фазового превращения.

\section{1. Введение}

Повышение рабочего давления за счет применения высокопрочных сталей, способных работать в сложных климатических условиях, является одним из наиболее перспективных путей повышения экономической эффективности магистральных трубопроводов [1-4]. Очевидным преимуществом применения высокопрочных труб является снижение металлоемкости (толщины стенки трубы) $[2,5,6]$. Прорывное улучшение конструктивной прочности низколегированных трубных сталей произошло в середине 70-х годов 20-го века в связи с разработкой и внедрением в промышленное производство контролируемой термомеханической обработки (TMCР - thermo-mechanical controlled processing), сочетающей в себе контролируемую прокатку и последующее контролируемое охлаждение [2, 6-9]. Следует отметить, что промышленное применение ТМСР в России вышло на новый уровень в 21 веке с запуском специализированных прокатных станов 5000 на ПАО «Северсталь» (г. Колпино), ПАО «ВМЗ» (г. Выкса) и ПАО «ММК» (г. Магнитогорск).

Переход от классов прочности Х52-Х65 к X70-X80 (К60-К65) и выше к X100 ( К80) ознаменовал переход от феррито-перлитных микроструктур к структурам с преобладанием продуктов сдвигового превращения (в основном бейнита), образование которых обеспечивалось контролем устойчивости переохлажденного аустенита за счет легирования и применением ускоренного охлаждения [2,8-11].

Новый уровень производства изделий с заданными функциональными свойствами и прогнозируемыми структурами требует управления параметрами технологических процессов в соответствии с кинетикой протекающих в материале физических процессов [12-14]. Важнейшими характеристиками процессов структурных и фазовых превращений, протекающих в материале при его обработке, являются величины их тепловых эффектов. Удельные величины тепловых эффектов, наряду с технологическими параметрами, определяют температуру материала в различные моменты его продвижения по технологической цепочке.
Целью данной работы являлось определение величины теплового эффекта бейнитного превращения в низкоуглеродистой трубной стали, необходимого для моделирования и, соответственно, управления ТМСР на современных прокатных станах.

\section{2. Материалы и методика исследований}

Для моделирования кинетики теплофизических процессов, протекающих в стали при скоростях охлаждения, воспроизводящих технологические параметры обработки, ИТЦ «Аусферр» разработал стенд (Рис. 1), состоящий из нагревательной печи с механизмом извлечения и фиксации образцов, блока измерения температуры, узла воздушного охлаждения с пневмосистемой, узла управления и регистрации информации. Вертикальная печь с цилиндрическим муфелем управляется независимым контроллером, поддерживающим заданную температуру. Для уменьшения окисления образцов в печь производится поддув аргона. Установка снабжена механизмом, позволяющим быстро извлекать образец из печи и фиксировать его в рабочей зоне установки. Привод механизма - ручной, механизм снабжен концевым выключателем, запускающим процедуру регистрации.

Для измерения температуры применяются три пирометра. Два из них - Raytek Marathon FR1A - двухрежимные пирометры ближнего инфракрасного спектра, имеющие диапазон измерения $550-1100^{\circ} \mathrm{C}$, третий быстродействующий пирометр OPTRIS CTfast, работающий в среднем ИК-диапазоне $\left(50-775^{\circ} \mathrm{C}\right)$. Перед измерением пирометры юстируются в точку, находящуюся в центре образца, извлеченного из печи. С этой же точкой совмещаются оси охлаждающих струй воздуха.

При пирометрических измерениях основная погрешность измерения определялась тем, что был неизвестен коэффициент черноты диагностируемой поверхности. В применявшейся методике эта проблема решена тем, что один из пирометров FR1A работает в режиме спектральных отношений. Показания пирометра спектральных отношений, работающего на близких спектральных линиях, не зависят от коэффициента черноты. Это 
позволяет откалибровать показания двух оставшихся пирометров непосредственно в эксперименте, и, тем самым, реализовать измерения температуры высокой точности в диапазоне $50-1100^{\circ} \mathrm{C}$. Все три пирометра включены в режим с токовым выходом. Регистрация токового сигнала производится с помощью узла управления и регистрации информации.

Обдув образцов производится «плоским» потоком воздуха под углом $15-30^{\circ}$ к образцу для исключения теневых зон. Пневмосистема стенда обеспечивает поддержание стабильного давления в магистралях в течение всего эксперимента. Принудительное охлаждение образцов производится с помощью блока сопел, имеющего 10 отверстий. Такая геометрия блока сопел позволяет получить однородный, близкий к плоскому, поток воздуха в зоне образца. Кроме блоков сопел пневмосистема стенда включает в себя компрессор с ресивером, редуктор, электромагнитный клапан, сенсоры для измерения давления.

Регистрация информации и управление установкой в процессе эксперимента проводилась специализированным вычислительным узлом, работающим под управлением ОС реального времени. Основная экспериментальная информация - показания пирометров. Кроме того, контролировалось давление в пневмосистеме и срабатывание концевого датчика. К каждому измерению система привязывает его время, что позволяет совмещать показания датчиков разных измерительных каналов.

В ходе эксперимента образец устанавливается в механизме перемещения и фиксации. С помощью узла юстирования все пирометры фокусируются в центральную точку поверхности образца. С этой же точкой совмещаются оси охлаждающих струй воздуха. Далее образец перемещается в печь, где нагревается до заданной температуры. После завершения выдержки происходит возвращение образца в первоначальное положение. Срабатывание концевых датчиков запускает процессы воздушного обдува и регистрации показаний пирометров.

В качестве материалов для исследований использовались образцы, вырезанные из промышленного листа малоуглеродистой низколегированной трубной стали типа 06Г2МБ ( 0.05 мас. $\% \mathrm{C}, \leq 2.0$ мас. $\% \mathrm{Mn}, \sim 0.2$ мас. $\% \mathrm{Mo}$, $\sim 0.05$ мас. \% Nb, остальное железо и неизбежные примеси), предназначенного для производства труб большого диаметра классов прочности К60, К65. Образцы представляли собой пластины $20 \times 70$ мм, толщина которых варьировалась в диапазоне от 0.25 до 1.10 мм. Подобные форма и размеры образцов обеспечивали теплообмен с внешней средой преимущественно в одном направлении, нормальном к плоскости поверхности пластин.

Образцы нагревали в печи стенда до температуры аустенитизации $980^{\circ} \mathrm{C}$ (без растворения карбидных фаз, выделившихся при промышленной ТМСР) и выдерживали в течении 30 минут. Скорость охлаждения образцов регулировали толщиной образцов и давлением в системе воздушного охлаждения.

Механическая подготовка образцов проводилась на комплексе пробоподготовки фирмы «Buehler» с использованием методики «New Buehler Conception».

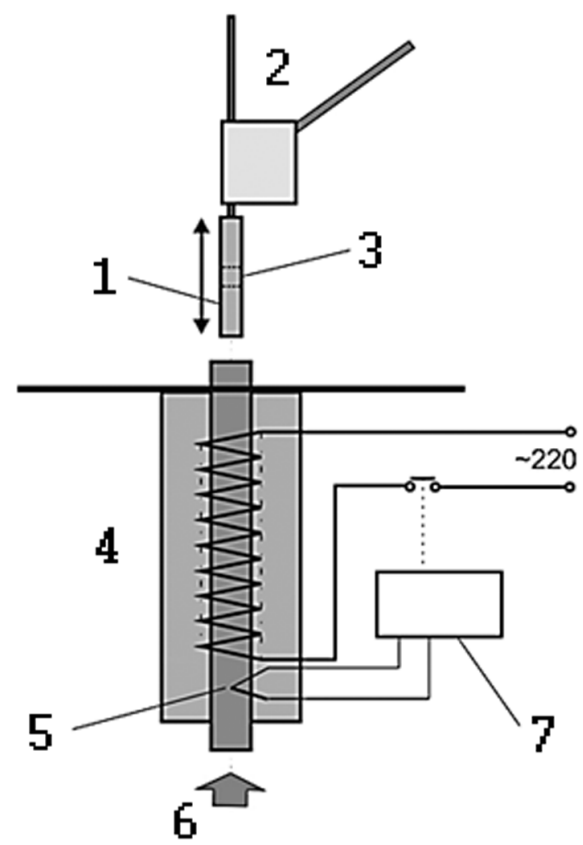

Рис. 1. Лабораторный стенд для теплофизических измерений: 1 - образец, 2 - механизм перемещения образца, 3 - область образца для измерений температуры, 4 - шахтная печь, 5 - термопара, 6 - подача аргона, 7 - контроллер печи.

Fig. 1. Laboratory bench for thermophysical measurements (schematic): 1 - sample, 2 - sample moving mechanism, 3 - the area of a sample for temperature measurements, 4 - shaft furnace, 5 - thermocouple, 6 - argon supply, 7 - furnace controller,

Металлографические исследования структуры проводились на структурном анализаторе, включающем в себя световой инвертированный металлографический микроскоп Nikon EPIPHOT-TME, цифровую камеру PixeLINK и анализатор SIAMS 600 с программными модулями для определения объемных долей структурных составляющих, размеров зерен и оценки карбидной неоднородности. Исследования микроструктуры проводили на микрошлифах после травления в реактиве Ниталь при оптических увеличениях $\times 400$ и $\times 1000$.

\section{3. Результаты исследования и их обсуждение}

В результате проделанных экспериментов были получены зависимости температуры образцов от времени при различных скоростях охлаждения (Рис. 2). При всех режимах обработки в образцах были зафиксированы практически идентичные структуры (Рис. 3), дисперсность которых несколько возрастала с увеличением скорости охлаждения. Согласно данным работ [15-17], посвященным, в том числе термокинетике фазовых переходов в малоуглеродистых сталях, данные микроструктуры сформировались преимущественно в результате бейнитного превращения. Цифровые представления термокинетических зависимостей, приведенных на Рис. 2, использовались для определения тепловых параметров фазового превращения.

Теплотехнические расчеты осуществлялись в предположении, что выравнивание температуры по толщине 
образца происходило мгновенно. Уравнение теплового баланса для однофазного образца имеет вид: $d H=\delta q_{r}+\delta q_{c}$ (где $d H$ - изменение энтальпии образца; $\delta q_{r}$ и $\delta q_{c}-$ эффекты радиационного и конвективного теплообмена). Данное уравнение в явном виде записывается как:

$$
(S / 2) \cdot h \cdot \rho \cdot C(T) d T=-S \cdot \varepsilon \cdot \sigma \cdot T^{4} d \tau-\alpha \cdot S \cdot\left(T-T_{o}\right) d \tau,
$$

где $T$ и $T_{o}$ - температуры образца и окружающей среды (охлаждающего воздуха); $C(T)$ - удельная теплоемкость, $\varepsilon$ и $\sigma$ коэффициенты черноты и Стефана-Больцмана, $\alpha-$ коэффициент теплоотдачи, $S$ и $h-$ площадь поверхности (без учета кромки) и толщина пластины, $\rho$ - плотность материала.

Поскольку конвективный эффект значительно превосходит радиационный, первым слагаемым в (1) можно пренебречь. Тогда в приближении постоянной удельной теплоемкости получается линейное дифференциальное уравнение

$$
d T / d \tau=-2 \cdot \alpha /(h \cdot \rho \cdot C) \cdot\left(T-T_{o}\right) .
$$

Это уравнение можно считать однородным, если $T_{o}$ принять за ноль температуры. Решение уравнения (2) хорошо известно:

$$
T(\tau)=\left(T_{N}-T_{o}\right) \cdot \exp (-\beta \cdot \tau)+T_{o},
$$

где $\beta=2 \cdot \alpha /(h \cdot \rho \cdot C)$ - декремент охлаждения, $T_{N}-$ начальная температура образца.

Участки экспериментальной кривой охлаждения $\rho$ (Рис. 4), за исключением температурного интервала фазовых превращений, хорошо описываются экспоненциальными функциями: $T_{\gamma}=927.7 \cdot \exp (-0.1135 \cdot \tau)+24$ для верхнего участка кривой $\rho$ и $T_{\alpha}=1444.9 \cdot \exp (-0.1217 \cdot \tau)+24$ для её нижнего участка.

Следует отметить, что температура начала охлаждения $\alpha$-фазы, полученная интерполяцией её кривой к $\tau=0$, попадает в $\delta$ область (Рис. 4 ).

Когда в образце протекает фазовое превращение в уравнении теплового баланса (1) появляется дополнительное слагаемое $\delta q_{t r}$, описывающее изменение энтальпии образца за счет внутреннего тепловыделения. В результате уравнение (2) становится существенно неоднородным:

$$
d T / d \tau=-\beta \cdot\left(T-T_{o}\right)+\phi(\tau)
$$

где $\phi(\tau)=\Phi(\tau) /(V \cdot \rho \cdot C), V-$ объем образца, $\Phi(\tau)-$ скорость выделения тепла в нем при фазовом превращении. Решение уравнения (4) имеет вид:

$$
\begin{aligned}
T(\tau) & =\left(T_{N}-T_{o}\right) \cdot \exp (-\beta \cdot \tau)+T_{o}+ \\
& +\exp (-\beta \cdot \tau) \cdot \int_{0}^{\tau} \phi\left(\tau^{\prime}\right) \exp \left(\beta \cdot \tau^{\prime}\right) d \tau^{\prime} .
\end{aligned}
$$

В отличие от (3) решение (5) имеет скорее эвристическое значение, поскольку функция $\Phi(\tau)$ неизвестна. По существу, необходимо решать обратную задачу поиска этой функции по известной функции $T(\tau)$. При этом необходимо учесть, что декремент охлаждения $\beta$ в интервале фазового превращения, где $\Phi(\tau)$ отлична от нуля, становится переменной величиной, зависящей от соотношения фаз. Поскольку $T(\tau)$ задана в цифровом виде, прямое аналитическое решение обратной задачи невоз- можно. В данной работе интересовала величина полного теплового эффекта $Q_{t r}$ при полиморфном $\gamma \rightarrow \alpha$ переходе в низкоуглеродистых трубных сталях для различных скоростей охлаждения, т. е. интеграл от $\Phi(\tau)$ по временному интервалу превращения.

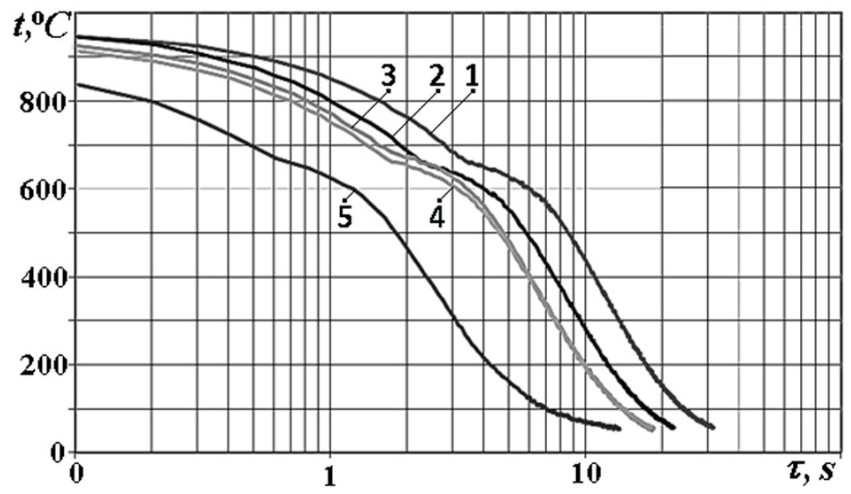

Pис. 2. Изменения температуры образцов при различных скоростях их охлаждения: 1 - режим № $1 ; 2-$ № $2 ; 3-$ № 3 ; $4-$ № $4 ; 5$ - № 5 .

Fig. 2. Changes in temperature of samples at different cooling rates: 1 - Mode No. 1; 2 - No. 2; 3 - No. 3; 4 - No. 4; 5 - No. 5 .

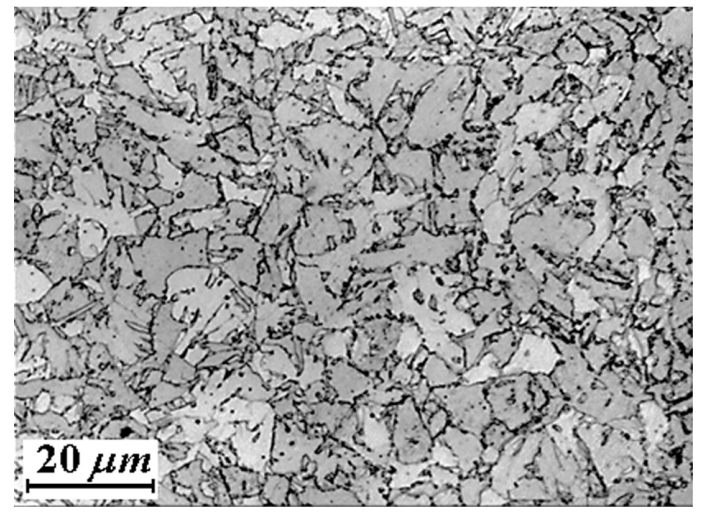

Рис. 3. Микроструктура образца, охлажденного по режиму № 1. Fig. 3. Microstructure of the sample cooled in regime No. 1.

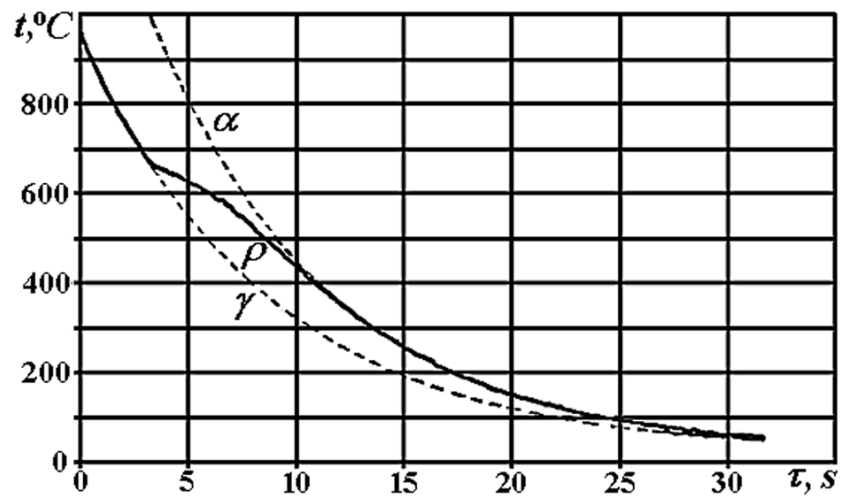

Pис. 4. Экспериментальная кривая охлаждения $-\rho$ (реальная температура образца) для режима №1. Экспоненциальные аппроксимации ее высоко $-\gamma$, и низкотемпературной $-\alpha$ частей.

Fig. 4. Experimental cooling curve $-\rho$ (real sample temperature) for mode No. 1. Exponential approximations of its high-temperature $-\gamma$ and low-temperature $-\alpha$ parts. 


\section{4. Определение теплового эффекта $\gamma \rightarrow \alpha$ превращения}

При протекании $\gamma \rightarrow \alpha$ превращения температура образца изменяется вследствие отвода тепла с его поверхности и выделения тепла в объеме образца $-\Delta Q_{t r}$. Вычислительная процедура определения $\Delta Q_{t r}$ организовывалась следующим образом.

Превращение протекало в интервале от $\tau_{0}$ до $\tau_{k}$. Принималось, что $\tau_{0}=0$ и $\tau_{k}=\tau_{t r}$, где $\tau_{t r}-$ длительность превращения. Начальная температура образца $T_{N}$ совпадала с температурой начала превращения. Расчет производился с шагом по времени $\Delta \tau=0.01$ с. Количество итераций в процедуре $-n=\tau_{t r} / \Delta \tau$.

С учетом близости $\beta_{\alpha}$ и $\beta_{\gamma}$, для переменного в интервале превращения декремента охлаждения $\beta$ принималась линейная аппроксимация $\beta=\beta_{\gamma} \cdot\left(\tau_{t r}-\tau\right) / \tau_{t r}+\beta_{\alpha} \cdot \tau / \tau_{t r}$. $\mathrm{B}$ рамках дискретной процедуры шаг изменения декремента охлаждения $\Delta \beta=\left(\beta_{\alpha}-\beta_{\gamma}\right) / n$. Подобная линейная аппроксимация принималась и для теплоемкости образца в двухфазной области.

Для режима № $1 \tau_{t r}=8.61 \mathrm{c}, T_{N}=673^{\circ} \mathrm{C}$, (Табл. 1), $\beta_{\gamma}=-0.1135 \mathrm{c}^{-1}, \beta_{\alpha}=-0.1217 \mathrm{c}^{-1}$. Теплоемкости $\gamma$ - и $\alpha$-фаз в [Дж/(кг $\left.\left.{ }^{\circ} \mathrm{C}\right)\right]$, согласно [18], задавались выражениями: $C_{\gamma}=0.1202 T+514.5 ; C_{\alpha}=0.4832 T+434.6$.

На первом шаге расчета $i=1$ изменяли температуру согласно (3): $T_{1}=\left(T_{N}-T_{o}\right) \cdot \exp \left(-\beta_{0} \cdot(\tau+\Delta \tau)\right)+T_{o}$, где $\beta_{0}=\beta_{\gamma}$. Теплоемкость образца $C_{1}$ при температуpe $T_{1}$ и его перегрев $\Delta T_{1}$ определялись из выражений: $C_{1}=C_{\gamma} \cdot(1-1 / n)+C_{\alpha} / n ; \Delta T_{1}=T_{R 1}-T_{1}$ (где $T_{R 1}-$ реальная (экспериментальная) температура образца (Рис. 5).

табл. 1. Параметры фазовых превращений.

Table 1. Parameters of phase transitions.

\begin{tabular}{|c|c|c|c|c|c|}
\hline No & $\begin{array}{c}d T / d \tau, \\
{ }^{\circ} \mathrm{C} / \mathrm{s}\end{array}$ & ${ }^{t}{ }^{\circ t r}{ }^{\circ}$ & ${ }^{t}{ }_{t r}{ }^{\circ} \mathrm{C}$ & $\begin{array}{c}\tau_{t r}, \\
\mathrm{~s}\end{array}$ & $\begin{array}{r}Q_{t}, \\
\mathrm{~kJ} / \mathrm{kg}\end{array}$ \\
\hline 1 & 110 & 673 & 366 & 8.6 & 112 \\
\hline 2 & 166 & 662 & 337 & 6.6 & 114 \\
\hline 3 & 193 & 727 & 324 & 6.0 & 124 \\
\hline 4 & 202 & 671 & 367 & 4.8 & 118 \\
\hline 5 & 449 & 692 & 494 & 1.3 & 108 \\
\hline
\end{tabular}

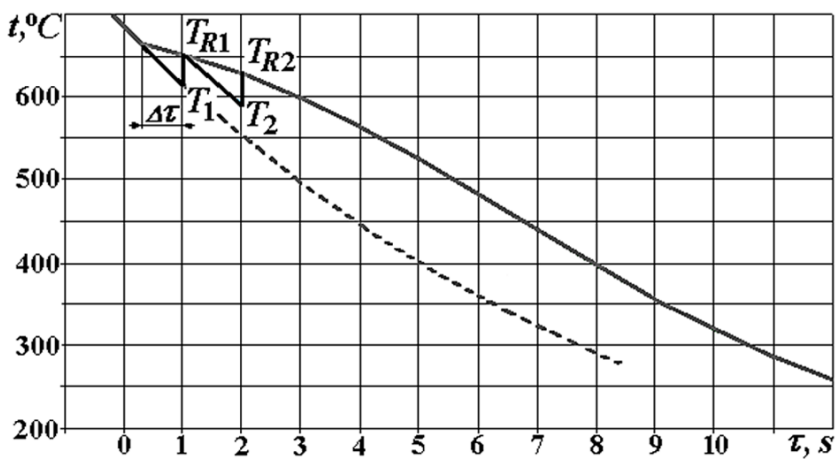

Рис. 5. Схема организации вычислительной процедуры определения $Q_{t r}$.

Fig. 5. Schematic illustration of the procedure for calculating the value $Q_{t r}$.
Теплота, выделившаяся в результате превращения, для первого шага расчета вычислялась как: $\Delta Q_{t r}=C_{1} \cdot \Delta T_{1}$. Для $i$-го шага расчетной процедуры: $\beta_{i-1}=\beta_{\gamma}+\Delta \beta \cdot(i-1)$; $T_{i}=\left(T_{R i-1}-T_{o}\right) \cdot \exp \left(-\beta_{i-1} \cdot \Delta \tau\right)+T_{o} ; \quad C_{i}=C_{\gamma} \cdot(1-i / n)+C_{\alpha} \cdot(i / n) ;$ $\Delta T_{i}=T_{R i}-T_{i} ; \quad \Delta Q_{i}=C_{i} \cdot \Delta T_{i}$. Вычисления продолжались до момента времени $\tau_{0}=\tau_{k}$, то есть до $i=n$. Суммарный тепловой эффект превращения $Q_{t r}$ определялся как: $Q_{t r}=\sum \Delta Q_{i}$.

Результаты определения термокинетических параметров, а именно: скорость охлаждения в интервале $950-850^{\circ} \mathrm{C}(d T / d \tau)$, температуры начала $\left(t_{0 t r}\right)$ и завершения $\left(t_{t r}\right)$ фазовых превращений, их длительность $\left(\tau_{t r}\right)$ и расчетные тепловые эффекты при различных $d T / d \tau$ приведены в Табл. 1. Очевидно, что погрешности определения, как тепловых эффектов, так и эффективных температур (в особенности температуры завершения превращения) возрастают с увеличением скорости охлаждения образцов. Важно отметить близость, как температур начала превращения, так и величин тепловых эффектов, зафиксированных для сравнительно большой вариации скоростей охлаждения. Это свидетельствует о практически атермическом характере бейнитного превращения в малоуглеродистых низколегированных сталях.

Подчеркнем, что тепловой эффект бейнитного превращения, зафиксированный в данной работе ( 120 кДж/кг), приблизительно в два раза превышает тепловой эффект мартенситного превращения для низкоуглеродистых сталей, рассчитанный как в известных [19], так и измеренный в современных исследованиях $[20]$.

\section{5. Выводы}

На разработанном оригинальном лабораторном стенде, позволяющем моделировать технологические скорости охлаждения горячекатаных листов в процессе ТМСР, определены термокинетические параметры и тепловые эффекты бейнитного превращения в малоуглеродистой низколегированной трубной стали марки 06Г2МБ. В интервале скоростей охлаждения 100 - 500 K/с температура начала бейнитного превращения составляет $660-730^{\circ} \mathrm{C}$, а величина теплового эффекта приблизительно равна 120 кДж/кг. Зафиксирован атермический характер бейнитного превращения, протекающего в процессе контролируемой термомеханической обработки.

Благодарности/Acknowledgements. Работа выполнена на оборудовании ОАО «Аусферр». Работа выполнена в рамках государственного задания ФАНО России, тема "Структура» ("Structure"), номер госрегистрации 0120146333. Авторы выражают признательность за содействие программе поддержки ведущих университетов РФ в иелях повышения их конкурентоспособности № 211 Правительства РФ №02.A03.21.0006./The study was performed on the equipment from OAO "Ausferr". The study was performed within the framework of the state assignment from the Federal Agency for Scientific Organizations of Russian Federation, theme "Structure", registration number 0120146333. The authors express their gratitude 
for the assistance to the program of support for the leading universities of the Russian Federation in order to improve their competitiveness No. 211 of the Government of the Russian Federation No. 02.A03.21.0006.

\section{Литература/References}

1. Yu.D. Morozov, M.Yu. Matrosov, S. Yu. Nastich, A. B. Arabei. Metallurgist. 7-8(52), 450 (2008). DOI: $10.1007 / \mathrm{s} 11015-008-9080-5$

2. D. J. Stolheim. Metallurgist. 11(55), 53 (2013). (in Russian) [Д. Дж. Столхейм. Металлург. 11(55), 53 (2013).]

3. V.I. Il'Inskii, P.P. Stepanov, L.I. Efron, S. V Golovin, V.V. Geier, M.Y. Matrosov, E.A. Goli-Oglu, O.P. Talanov. Metallurgist. 1-2(58), 38 (2014). DOI: 10.1007/s11015-014-9865-7

4. S. Y. Nastich, Y. D. Morozov, V.L. Kornilov, S. V. Denisov, M. A. Molostov. Steel in Translation. 5(39), 431 (2009). DOI: 10.3103/S0967091209050179

5. Cl. Petersen, K. Corbett, D. Fairchild, Sc. Papka, M. Macia. Improving long-distance gas transmission economics. XI20 development over-view: Proceedings of 4th International Pipeline Conference. Ostend (2004), P. 3-29.

6. K. Hulka, P. Peters, F. Haisterkamp. Stall. 10, 62 (1997). (in Russian) [К. Хулка, Ф. Хайстеркамп. Сталь. 10, 62 (1997).]

7. Yu. D. Morozov, S. Yu. Nastich, M.Yu. Matrosov, O.N. Chevskaya. Metallurgist. 1-2(58), 21 (2008). DOI: $10.1007 /$ s11015-008-9003-5

8. M. Yu. Matrosov, A. A. Kichkina, A. A. Efimov, L. I. Éfron, O.A. Bagmet. Metallurgist. 7-8(51), 367 (2007). DOI: 10.1007/s11015-007-0068-3

9. S. Y. Nastich, Y.D. Morozov, M. Y. Matrosov, S. V. Denisov, V.V. Galkin, P.A. Stekanov. Metallurgist. 11-12, 810 (2012). DOI: 10.1007/s11015-012-9507-X

10. A. B. Arabey. Steel in Translation. 7(40), 601 (2010). DOI: $10.3103 /$ S0967091210070016
11. I. Pyshmintsev, V.I. Stolyarov, A.M. Gervas'ev et al. Science and technology in the gas industry. 1, 56 (2009). (in Russian) [И.Ю. Пышминцев, В.И. Столяров, A. М. Гервасьев и др. Наука и техника в газовой промышленности. 1, 56 (2009).]

12. E. A. Goli-Oglu, Yu.D. Morozov, L.I. Éfron. Metal Science and Heat Treatment. 5-6(55), 294 (2013). DOI: $10.1007 /$ s1 1041-013-9622-8

13. I.Y. Pyshmintsev, A.O. Struin, A.M. Gervasyev, at al. Metallurgist. 3-4(60), 405 (2016). DOI: $10.1007 / \mathrm{s} 11015-016-0306-7$

14. E. A. Goli-Oglu, Y.D. Morozov, L.I. Efron. Steel in Translation. 2(43), 113 (2013). DOI: $10.3103 /$ S0967091213020101

15. L. Rancel, M. Gómez, S.F. Medina, I. Gutierrez. Materials Science and Engineering A. 530, 21 (2011). DOI: 10.1016/j.msea.2011.09.001

16. J. Cao, J. Yan, J.Yu. T. Zhang. Materials Science \& Engineering A. 639, 192 (2015). DOI: 10.1016/j.msea.2015.05.005

17. A. Grajcar, W. Zalecki, P. Skrzypczyk at al. J. Therm Anal Calorim. 118, 739 (2014). DOI: $10.1007 / \mathrm{s} 10973-014-4054-2$

18. D. A. Ivanov, N. V. Kuvayev, T. V. Kuvayeva. Teoriya i praktika metallurgii. 1-2, 43 (2010). (in Russian) [Д.А. Иванов, Н.В. Куваев, Т.В. Куваева. Теория и практика металлургии. 1 - 2, 43 (2010).]

19. G. I. Sufiyanova, L. V. Spivak. Fazovye prevrashcheniya nizkouglerodistykh martensitnykh stalyakh: Proceedings "Fizika dlya Permskogo kraya". Perm (2016) P. 37 - 39. (in Russian) [Г.И. Суфиянова, Л.В. Спивак. Фазовые превращения низкоуглеродистых мартенситных сталях: Материалы региональной научно-практической конференции студентов, аспирантов и молодых ученых "Физика для Пермского края". Пермь (2016) C. 37 - 39.]

20. H.K.D.H. Bhadeshia. Journal de Physique IV Collogue. 7 , C5-367-C5-376 (1997). 